\title{
Impacto de intervenção psicoeducacional na autoeficácia percebida de estudantes de enfermagem
}

\author{
Impact of psychoeducational intervention on nursing students' perceived self-efficacy
}

Impacto de intervención psicoeducacional en la autoeficacia percibida de estudiantes de enfermería

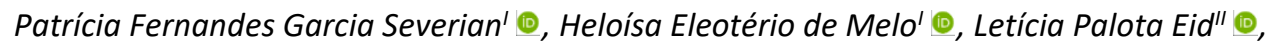

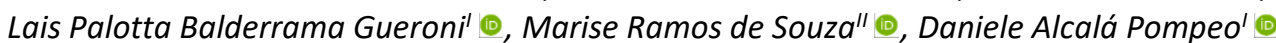

'Faculdade de Medicina de São José do Rio Preto, São José do Rio Preto, SP, Brasil; "Universidade Federal de Jataí, Jataí, GO, Brasil

\begin{abstract}
RESUMO
Objetivo: avaliar o impacto de um programa psicoeducacional nos níveis de autoeficácia, autoestima e sintomas ansiosos e depressivos em estudantes no início da graduação em enfermagem. Método: pesquisa multicêntrica, quase experimental, do tipo tempo-série, desenvolvida em duas instituições de ensino superior públicas, com 82 estudantes, no período de setembro de 2018 a maio de 2019. O programa psicoeducacional foi elaborado com base no construto da autoeficácia, baseando-se nas atividades de enfermagem da intervenção "Fortalecimento da autoestima", proposta pela Nursing Interventions Classification Os desfechos avaliados foram: autoeficácia, mensurada pela Escala de Autoeficácia Geral e Percebida; autoestima, mensurada pela Escala de Autoestima de Rosenberg; sintomas ansiosos e depressivos, avaliados pela Escala Hospitalar de Ansiedade e Depressão. Resultados: a intervenção psicoeducacional teve efeito positivo na percepção de autoeficácia geral dos estudantes. A autoestima e os sintomas ansiosos e depressivos não foram influenciados. Conclusão: os níveis de autoeficácia foram positivamente impactados pelo programa implementado.
\end{abstract}

Descritores: Promoção da Saúde; Saúde Mental; Autoeficácia; Estudantes de Enfermagem.

\begin{abstract}
Objective: to evaluate the impact of a psychoeducational program on the levels of self-efficacy, self-esteem, and anxious and depressive symptoms in students starting their undergraduate nursing program. Method: this quasi-experimental, multicenter, time-series type study was conducted with 82 students at two public institutions of higher education, from September 2018 to May 2019. Based on the self-efficacy construct, the psychoeducational program contemplated nursing activities of the "Strengthening self-esteem" intervention proposed by the Nursing Interventions Classification. The outcomes evaluated were self-efficacy, on the General and Perceived Self-Efficacy Scale; self-esteem, on the Rosenberg Self-Esteem Scale; and anxious and depressive symptoms, on the Hospital Anxiety and Depression Scale. Results: the psychoeducational intervention had a positive effect on the students' perception of general self-efficacy. Self-esteem and anxious and depressive symptoms were not influenced. Conclusion: the program impacted levels of self-efficacy positively.
\end{abstract}

Descriptors: Health promotion; Mental health; Self-efficacy; Nursing students.

\section{RESUMEN}

Objetivo: evaluar el impacto de un programa psicoeducativo en los niveles de autoeficacia, autoestima y síntomas ansiosos y depresivos en estudiantes al inicio de sus estudios universitarios en enfermería. Método: investigación multicéntrica, cuasiexperimental, tipo serie temporal, desarrollada en dos instituciones públicas de educación superior, con 82 estudiantes, de septiembre de 2018 a mayo de 2019. El programa psicoeducativo se desarrolló con base en el constructo de autoeficacia y las actividades de enfermería de la intervención "Fortalecimiento de la autoestima", propuesta por la Nursing Interventions Classification. Los resultados evaluados fueron: autoeficacia, medida por la Escala de Autoeficacia General y Percibida; autoestima, medida por la Escala de Autoestima de Rosenberg; síntomas de ansiedad y depresión, evaluados por la Escala Hospitalaria de Ansiedad y Depresión. Resultados: la intervención psicoeducativa tuvo un efecto positivo sobre la percepción de autoeficacia general de los estudiantes. La autoestima y los síntomas de ansiedad y depresión no se vieron afectados. Conclusión: los niveles de autoeficacia fueron impactados positivamente por el programa implementado.

Descriptores: Promoción de la salud; Salud mental; Autoeficacia; Estudiantes de enfermería.

\section{INTRODUÇÃO}

Ingressar na universidade representa para os estudantes algo empolgante, estimulante, desafiador e a concretização de um sonho. Apesar disso, a população universitária tem sido preocupação constante das comunidades acadêmicas e científicas de todo o mundo ${ }^{1,2}$

A transição do ensino médio para o superior coincide com um período crítico de desenvolvimento biológico, psicológico e social acelerado. Essa transição acontece em um momento sensível do ciclo da vida, comumente marcado pela separação da família, desenvolvimento de novas conexões sociais, autonomia, responsabilidade e alterações emocionais, tornando-se um período de pico de risco para o aparecimento de doenças mentais graves e persistentes ${ }^{1}$.

Agradecimentos à Coordenação de Aperfeiçoamento de Pessoal de Nível Superior (CAPES), código de financiamento 001 Autora correspondente: Daniele Alcalá Pompeo. E-mail: daniele.pompeo@famerp.br

Editora Científica: Cristiane Helena Gallasch; Editora Associada: Mercedes Neto 
Dentro deste contexto, a necessidade de cuidados efetivos, atrativos e acessíveis à saúde mental no âmbito acadêmico, que busquem otimizar a edificação de recursos mentais positivos, é fundamental e urgente. O sofrimento mental nessa fase pode comprometer o bem-estar dos alunos e o sucesso acadêmico, além da possibilidade de consequências irreversíveis à vida do estudante e ao ambiente da universidade, como evidenciado pelas recentes tragédias anunciadas em diferentes instituições de ensino do Brasil e no mundo².

Portanto, fortalecer os pilares da saúde mental, com foco na promoção da saúde, é a chave para implementação de ações fundamentadas no âmbito da prevenção primária de transtornos psíquicos, uma vez que potencializa subsídios mentais necessários para o enfrentamento assertivo diante de situações geradoras de estresse.

Neste cenário, evidências crescentes têm demonstrado a autoeficácia como sendo um dos principais pilares de sustentação da saúde mental em jovens universitários, associando-se positivamente com níveis de aprendizagem, desenvolvimento de responsabilidade e independência, sentimento de confiança, atendimento às necessidades do paciente e atitudes éticas ${ }^{3}$, além de estar relacionada diretamente com a autoestima e com o amortecimento de efeitos deletérios advindos do estresse $e^{4,5}$ e de sintomas depressivos e ansiosos ${ }^{4,6}$.

Em ampla busca na literatura, observa-se escassez de evidências científicas robustas sobre intervenções para promover saúde mental em universitários, sobretudo com foco no fortalecimento de habilidades, e não na melhora de doenças ou quadros de sofrimento já instalados. Nesse sentido, uma recente meta análise demonstrou que as intervenções com foco na promoção da saúde mental apresentaram efeito positivo no bem-estar mental de estudantes ${ }^{7}$. Entretanto, a conclusão foi limitada em razão do número reduzido de estudos encontrados.

À vista disso, um obstáculo fundamental para a implementação de estratégias baseadas em evidências tem sido a falta relativa de dados sistematicamente coletados para ajudar as universidades no desenvolvimento de um sistema coordenado e abrangente de atenção à saúde mental dos estudantes ${ }^{2}$. Assim, torna-se evidente a necessidade de ampliar as investigações acerca dessas intervenções que promovam e fortaleçam habilidades necessárias para suportar as adversidades da vida pessoal e acadêmica e, desta forma, evitar ou minimizar o adoecimento.

Pautando-se nestas lacunas, o objetivo dessa pesquisa foi avaliar o impacto de um programa psicoeducacional nos níveis de percepção de autoeficácia, autoestima e sintomas de ansiedade e depressão em estudantes no início da graduação em enfermagem.

\section{MÉTODO}

Pesquisa multicêntrica, quase experimental, do tipo tempo-série, desenvolvida com estudantes de graduação em enfermagem de duas instituições de ensino superior (IES) públicas, uma no estado de São Paulo e a outra em Goiás. A escolha dessas IES ocorreu pela localização geográfica divergente, facilidade de coleta de dados e vulnerabilidade mental identificada cotidianamente nesses estudantes, revelada pela busca expressiva de serviços de apoio psicológico oferecidos pelas referidas IES, por motivos que incluem principalmente o estresse, baixa tolerância à frustrações, ansiedade, depressão, baixo rendimento escolar, ideação e tentativa de suicídio.

A população foi constituída por todos os alunos matriculados no 10 ano de graduação em enfermagem das IES 1 $(n=60)$ e $2(n=30)$. A preferência pelo ingressante se justificou por ser um período de transição de ciclos, caracterizado por mudança de ambiente, de amigos, de rotinas e do aumento das responsabilidades e da autonomia.

Os critérios de inclusão estabelecidos foram: estar matriculado no 10 ano da graduação das IES 1 ou 2 , ter 18 anos ou mais e estar presente nas datas de intervenção e coleta de dados. Foram excluídos os estudantes que não haviam ingressado no ano corrente (reprovados ou retorno após período de trancamento de matrícula). A Figura 1 demonstra o processo de seleção e amostragem dos participantes.

Para verificar o perfil dos participantes, foi aplicado um Questionário de caracterização sociodemográfica ${ }^{8}$, com variáveis relacionadas à relação familiar, condição socioeconômica, satisfação com o curso, profissão, sobrecarga com as atividades acadêmicas, além de idade, sexo e estado civil.

Os desfechos foram avaliados antes e depois do programa psicoeducacional: I) percepção de autoeficácia, mensurada pela Escala de Autoeficácia Geral e Percebida (EAEGP), II) autoestima, avaliada pela Escala de Autoestima de Rosenberg (EAR), III) sintomas de ansiedade e depressão, determinados pela Escala Hospitalar de Ansiedade e Depressão, do inglês Hospital Anxiety and Depression Scale (HAD-S).

A EAFGP é composta por 10 itens, com respostas do tipo Likert que variam de um a cinco. Esses itens retratam o alcance de metas e à percepção do indivíduo perante o sucesso em uma situação. O valor da escala varia de 10 a 50 . Pontuação mais elevada indica maior percepção da autoeficácia. As propriedades psicométricas da Escala de Autoeficácia Geral e Percebida são satisfatórias em sua versão original (alfa de Cronbach de 0,84 ) e na de validação para o Brasil (alfa de Cronbach de 0,81), realizada entre estudantes universitários ${ }^{9}$. 


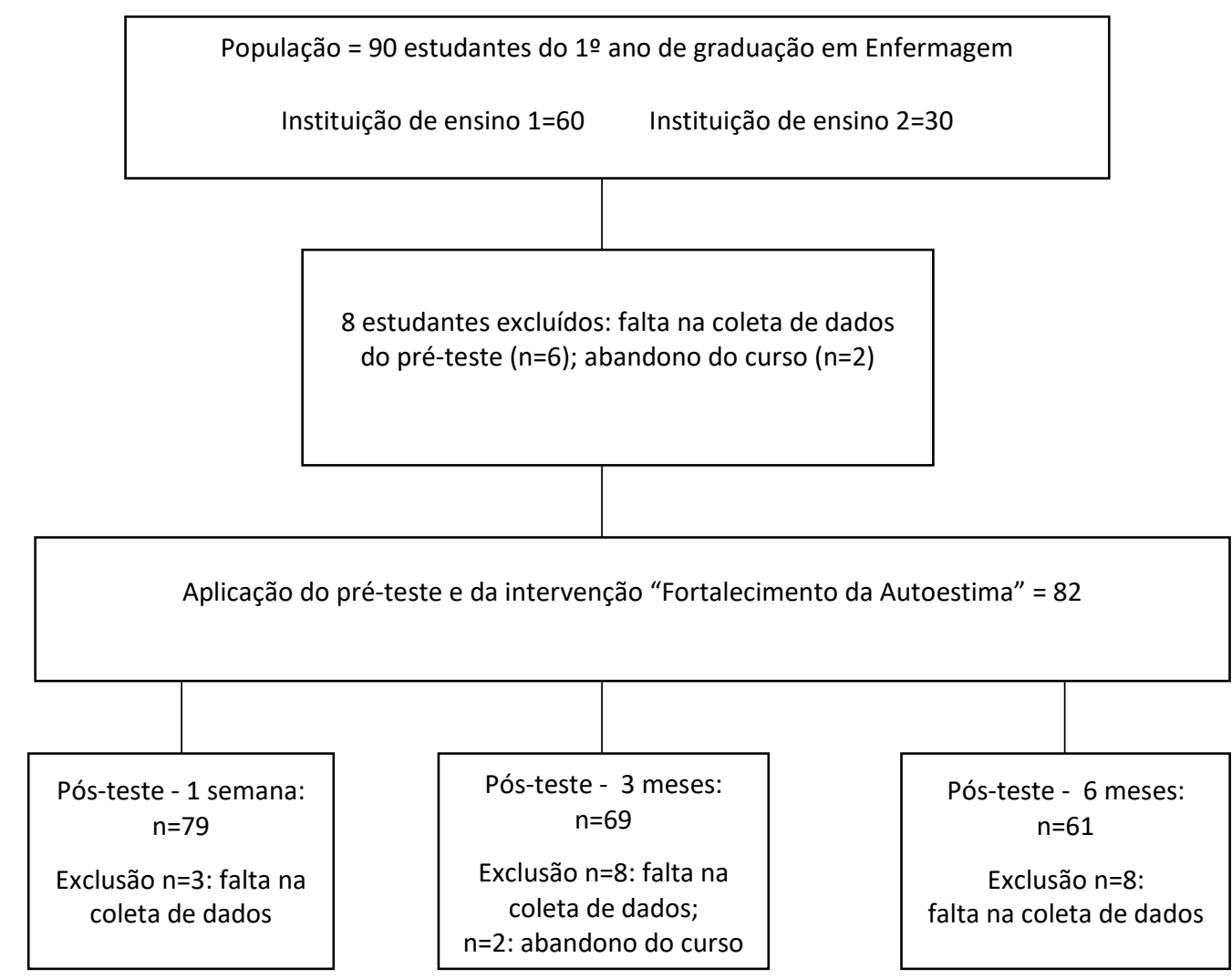

FIGURA 1: Processo de seleção e amostragem dos estudantes. São José do Rio Preto, SP, Jataí, GO, Brasil, 2019.

A EAR, validada para o Brasil, possui 10 itens, com respostas Likert, de quatro pontos. A medida da autoestima é obtida pela soma dos valores das respostas aos itens e pode variar de 10 a 40 . A autoestima é classificada em alta ou satisfatória (maior que 30 pontos), média (de 20 a 30 pontos) e baixa ou insatisfatória (menor que 20 pontos). Possui valores elevados de consistência interna (alfa de Cronbach: 0,90) ${ }^{10}$ e tem sido um dos instrumentos mais empregados na literatura nacional e internacional para avaliar a autoestima.

A HADS foi elaborada para avaliar sintomas ansiosos e depressivos em hospital clínico, mas posteriormente passou a ser empregada em outros contextos extra-hospitalares, incluindo pessoas sadias e estudantes do ensino superior ${ }^{11,12}$. Essa escala possui 14 questões intercaladas de sintomas ansiosos e depressivos e apresenta boa sensibilidade (70,8\% a $80,6 \%$ ) e especificidade (69,6\% a 90,9\%) quando comparada à Escala de Ansiedade de Beck e à Escala de Depressão de Beck, ambas consideradas padrão-ouro ${ }^{13}$. Cada item da escala contempla quatro respostas de 0 a 3 , totalizando uma pontuação máxima de 21 pontos para cada subescala. Foram considerados sem sintomas ansiosos e depressivos àqueles cuja pontuação variou de 0 a 8 pontos, em cada subescala. Os participantes com pontuação $\geq 9$ foram classificados com sintomatologia ansiosa e depressiva ${ }^{13}$.

O programa psicoeducacional teve o objetivo de melhorar a percepção de eficácia pessoal e autoestima de estudantes de graduação. Foi elaborado com base no construto da autoeficácia, presente na Teoria Social Cognitiva de Bandura $^{14}$. Esse programa baseou-se nas atividades de enfermagem da intervenção "Fortalecimento da autoestima", proposta pela Nursing Interventions Classification (NIC). A NIC define fortalecimento da autoestima como a assistência ao indivíduo para aumentar julgamento pessoal do próprio valor ${ }^{15}$.

O programa foi estruturado em oito sessões, de acordo com os objetivos a serem alcançados:

1. Autoconhecimento e autoestima: encorajar o autoconhecimento, a identificação de pontos fortes e as fragilidades, a verbalização de afirmativas positivas de si e o monitoramento de sentimentos de auto-negatividade.

2. Vencendo a autocrítica, o sentimento de culpa e a insegurança: auxiliar o estudante a encontrar autoaceitação, evitar críticas e identificar o impacto do grupo sobre seus sentimentos de valor próprio; explorar os motivos da autocrítica.

3. Autoconfiança e autoestima: auxiliar o estudante a determinar sua autoconfiança; reforçar pontos positivos e evitar críticas. 
4. Você como protagonista da própria vida: encorajar maior responsabilidade por si mesmo; auxiliar a avaliar o próprio comportamento e monitorar a falta de seguimento em alcançar metas; encorajar o estudante a aceitar novos desafios.

5. Planejamento de metas e pequenos desafios: auxiliar o estudante no estabelecimento de metas realistas; encorajar a avaliar o próprio comportamento, aceitar novos desafios e ter maior responsabilidade sobre si mesmo e sobre suas metas; relembrar experiências de sucesso que aumentem a autonomia.

6. Criação de uma boa visão de si: auxiliar na reavaliação da autopercepção negativa e na autoaceitação; ensinar a estratégia de recompensar e elogiar o progresso perante as metas alcançadas; encorajar a identificação de pontos fortes e o lócus de controle.

7. Evitar comparações: auxiliar a superar provocações e avaliar o próprio comportamento; transmitir confiança na capacidade de lidar com as situações.

8. Pensamento positivo é essencial: auxiliar a identificar respostas positivas de outras pessoas; explorar conquistas alcançadas e transmitir confiança na capacidade de lidar com as situações.

O programa foi realizado de forma presencial, em grupo e por meio da estratégia de educação em saúde. As atividades foram conduzidas pelos próprios pesquisadores, em sala de aula e com duração de 40 minutos. 0 conteúdo foi pautado em mensagens positivas, textos reflexivos e histórias de vida. A principal estratégia utilizada foi a persuasão verbal, em que os participantes foram levados, por meio de sugestões, a acreditar que são capazes de superar suas dificuldades e modificar seu comportamento. Para isso, o condutor utilizava palavras e frases de motivação (exemplos: desafios, oportunidades, enfrentar, confiança, metas, dentre outras). A palavra "você" foi utilizada, com frequência, como gatilho para ativar a persuasão, pois coloca a pessoa como cerne da situação.

Dois reforços foram oferecidos (o primeiro após a 4ạ sessão e o segundo após a 8a sessão), cujos objetivos foram esclarecer dúvidas, reforçar orientações recebidas nas intervenções e motivar os estudantes na realização das atividades propostas. Nessa etapa, além da persuasão, utilizaram-se a experiência direta de domínio (pela recordação de situações vivenciadas em outros momentos e encorajamento para experiências futuras), experiência vicária (pela escuta de relatos de outros participantes) e o feedback fisiológico (pela identificação das situações estressantes e auxílio de como gerenciá-lo) ${ }^{14}$.

As intervenções e os reforços foram realizados em intervalos de uma semana entre um encontro e outro, totalizando 10 semanas. O número de sessões e a periodicidade foram estabelecidos a partir de outros estudos que também testaram intervenções para melhora da saúde mental em estudantes de enfermagem ${ }^{4,16}$.

As coletas de dados e as intervenções de enfermagem foram realizadas pelos pesquisadores lotados em suas respectivas instituições de ensino, no período de setembro de 2018 a maio de 2019. O conteúdo abordado foi construído em conjunto e as pesquisadoras realizaram um treinamento teórico e prático para garantir a uniformidade na execução das atividades a serem desenvolvidas.

Os dados foram coletados em sala de aula e o participante respondeu aos instrumentos em sua carteira, sem a influência dos professores ou dos pesquisadores, com duração aproximada de 25 minutos. Após o preenchimento, os questionários respondidos foram colocados em envelope fechado. As mensurações pós-teste foram realizadas em dois momentos: uma semana e três meses após o segundo reforço.

Os dados foram processados e analisados por meio do programa Minitab 17 (Minitab Inc.). Foram realizadas análises descritivas para as variáveis de caracterização amostral. Para analisar as associações da autoestima e sintomas ansiosos e depressivos pré e pós-intervenção foi aplicado o Teste Exato de Fisher para duas proporções. O Teste KruskalWallis com posterior teste de comparação múltipla de Dunn foi empregado para verificar a diferença da autoeficácia nos períodos antes e após o programa psicoeducacional. Para a avaliação da normalidade dos dados foi aplicado o Teste de Anderson-Darling. O nível de significância aplicado para os testes foi de 0,05 ou $5 \%$.

O protocolo de pesquisa foi aprovado pelos Comitês de Ética em Pesquisa das instituições envolvidas, conforme regulamenta os dispositivos da Resolução no 466/2012.

\section{RESULTADOS}

Participaram desse estudo 82 estudantes do 10 ano da graduação em enfermagem. Parte expressiva deles era do sexo feminino $(71 ; 86,59 \%)$, não apresentava companheiro $(76 ; 92,68 \%)$ e pertencia a IES $1(52 ; 63,41 \%)$. A maior parte dos estudantes relatou morar com os pais $(39 ; 47,56 \%)$, ter relação familiar harmoniosa $(70 ; 85,37 \%)$ e possuir recursos financeiros suficientes para seu sustento $(50 ; 60,98 \%)$.

A maioria dos participantes afirmou que a profissão enfermagem não foi sua primeira opção de curso no vestibular (48; 59,26\%), sendo que destes 48 estudantes, 36 (73,47\%) desejariam ter cursado medicina. A maioria se diz satisfeita 
com a profissão $(76 ; 92,68 \%)$ e com o curso $(76 ; 92,68 \%)$ e não pensa em abandoná-la $(49 ; 59,76 \%)$. Do total de 82 estudantes, a maioria se sente sobrecarregada com as atividades da graduação (70; $85,37 \%)$ e não exerce trabalho remunerado $(76 ; 92,68 \%)$.

A idade média dos 82 estudantes avaliados foi de 19,89 anos, com desvio padrão de 3,94 anos e mediana de 19,00 anos. A idade mínima observada foi de 18,0 e a máxima de 39,0 anos.

A intervenção proposta impactou nos escores de autoeficácia até os três meses após a intervenção como demosntrado na Tabela 1.

TABELA 1: Estatísticas descritivas da autoeficácia de acordo com os períodos de análise ( $\mathrm{N}=61)$ São José do Rio Preto-SP/ Jataí-GO, Brasil, 2019.

\begin{tabular}{lclc}
\hline Autoeficácia percebida & Média \pm desvio padrão & Mediana $^{2}$ & Valor P1 \\
\hline Pré-intervenção & $31,75 \pm 7,54$ & $32,00^{\mathrm{b}}$ & \\
Pós-intervenção (1 semana) & $34,93 \pm 7,07$ & $35,00^{\mathrm{ab}}$ & 0,021 \\
Pós-intervenção (3 meses) & $36,08 \pm 6,47$ & $36,00^{\mathrm{a}}$ & \\
\hline 'Valor P referente ao teste de Kruskal-Wallis a P<0,05. ${ }^{2}$ Letras diferentes na mesma coluna \\
indicam diferenças significativas pelo teste de comparação múltipla de Dunn a P<0,05.
\end{tabular}

No entanto, esse efeito não foi verificado nos níveis de autoestima dos graduandos: comparação da fase pré-teste com o pós-teste uma semana $(p=1,000)$ e pós-teste três meses $(p=1,000)$. Ressalta-se que não houve diferença estatística nos escores de percepção da autoeficácia entre os participantes das IES 1 e 2 ( $P=0,453)$.

A Tabela 2 apresenta os escores de sintomas ansiosos e depressivos comparando os períodos avaliados no estudo.

Tabela 2: Percentuais do teste de duas proporções para as comparações entre os escores dos sintomas de ansiedade e depressão dos alunos, de acordo com os períodos pré e pósintervenção. São José do Rio Preto-SP/ Jataí-GO, Brasil, 2019.

\begin{tabular}{lccccc}
\hline \multirow{2}{*}{ Períodos avaliados } & \multicolumn{3}{c}{ Presença } & \multicolumn{3}{c}{ Ausência } & \multirow{2}{*}{ Valor $\mathbf{P}^{1}$} \\
\cline { 2 - 5 } & $\mathbf{N}$ & $\mathbf{\%}$ & $\mathbf{N}$ & $\%$ & \\
\hline Sintoma de Ansiedade (HADS-A) & & & & & \\
\hline Pré-intervenção & 42 & 68,85 & 19 & 31,15 & \\
Pós-intervenção (1 semana) & 33 & 54,10 & 28 & 45,90 & 0,136 \\
Pós-intervenção (3 meses) & 33 & 54,10 & 28 & 45,90 & 0,136 \\
\hline Sintoma de Depressão (HADS-D) & & & & & \\
\hline Pré-intervenção & 18 & 29,51 & 43 & 70,49 & \\
Pós-intervenção (1 semana) & 17 & 27,87 & 44 & 72,13 & 1,000 \\
Pós-intervenção (3 meses) & 12 & 19,67 & 49 & 80,33 & 0,293 \\
\hline
\end{tabular}

${ }^{1}$ Valor $\mathrm{P}$ referente ao teste exato de Fisher para duas proporções a $\mathrm{P}<0,05$.

Os resultados indicaram decréscimo dos sintomas ansiosos e depressivos nos períodos pós-intervenção, evidenciando melhora clínica desses sintomas. Apesar disso, testes comparativos para duas proporções demonstraram que não foram impactados pela intervenção testada $(P>0,05)$.

\section{DISCUSSÃO}

Os resultados desse estudo mostraram que a intervenção psicoeducacional teve efeito positivo na percepção de autoeficácia geral de estudantes de enfermagem, até três meses após a sua implementação. Já a autoestima e os sintomas ansiosos e depressivos não foram influenciados pela intervenção. A literatura que versa sobre programas ou ações que promovam, fortaleçam ou melhorarem a autoestima e a autoeficácia é escassa, limitando comparações.

Esse fato pode ser evidenciado pela recente publicação de revisão sistemática que demonstrou a necessidade de mais estudos que explorem ações para gerenciar a saúde mental de universitários de enfermagem. Os pesquisadores identificaram apenas 12 estudos envolvendo essa temática, que representaram 651 participantes. A maior parte das intervenções é direcionada a ansiedade, depressão e estresse. O estudo ainda concluiu que não foram observadas investigações que promovessem a autoeficácia nessa população ${ }^{17}$.

No entanto, foram identificados alguns estudos clínicos com resultados positivos em relação aos níveis de autoeficácia de universitários após intervenções. Um desses estudos verificou os efeitos de um programa de gerenciamento do estresse, pautado na terapia cognitivo-comportamental, em grupo, por 10 semanas. Os estudantes 
de enfermagem do grupo intervenção obtiveram maiores escores de autoeficácia e autoestima imediatamente após a realização do programa e na mensuração após um ano, quando comparados aos controles ${ }^{4}$.

Estudo semelhante, com estudantes do primeiro ano de um curso de medicina $(n=42)$, testou uma intervenção para o gerenciamento do estresse, estruturada na construção de habilidades de relaxamento, enfrentamento e nutrição, com duração de oito semanas. Depois da intervenção, observaram-se níveis de ansiedade reduzidos e eficácia pessoal elevada, quando comparados aos escores do pré-teste ${ }^{16}$.

Um programa de psicoterapia positiva em grupo, conduzido para proporcionar melhora da eficácia pessoal e da depressão, com duração de oito semanas, foi testado em estudantes de enfermagem com depressão de níveis leve e moderado $(n=76)$. Os resultados revelaram relação entre as variáveis, sugerindo que a psicoterapia positiva pode otimizar a autoeficácia e, desta forma, aliviar os sintomas depressivos, sendo recomendada como método de apoio psicológico 6 .

Verificou-se, por meio de estudos, que os graduandos com estresse puderam se beneficiar de intervenções para fortalecer a saúde mental ${ }^{5}$ e que a autoeficácia apareceu como um dos preditores mais importantes no controle e gerenciamento desse problema ${ }^{4,6,16}$.

Essa relação inversa entre estresse e autoeficácia também pode ser evidenciada em investigações na esfera profissional e acadêmica. Nesse sentido, um recente estudo italiano comprovou que a positividade, notas escolares e comportamentos de cidadania foram mediados por crenças de autoeficácia ${ }^{18}$. Além disso, pensamentos positivos sobre a confiança do indivíduo em sua própria capacidade contribuíram para manutenção de ótimos níveis de autoestima, uma vez que os indivíduos sentem que têm maior capacidade de enfrentar situações estressantes, sendo de suma importância sua abordagem em programas de saúde mental ${ }^{19}$.

A autoeficácia é considerada uma construção pessoal, classificada como um antecedente poderoso para o engajamento e o desempenho acadêmico e laboral, bem como um recurso amortecedor contra o Burnout ${ }^{18}$. Esses achados sugerem que intervenções focadas na autoeficácia e outras habilidades emocionais, durante a graduação, podem proteger futuros enfermeiros de esgotamento físico e emocional, além de proporcionar maior segurança e envolvimento no trabalho.

Ao contrário de alguns estudos anteriores, os sintomas ansiosos ${ }^{16,20}$ e depressivos ${ }^{6,20,21}$ não foram impactados pela intervenção executada. No entanto, apresentaram uma queda nas duas medidas após a intervenção. Embora estas oscilações não tenham sido significativas, traduzem uma melhora clínica desses sintomas após a exposição dos alunos ao programa implementado - nove estudantes que anteriormente haviam sido classificados com sintomas ansiosos e seis com depressivos apresentaram melhora. Da mesma forma, os estudantes da amostra demonstraram autoestima predominantemente moderada, que não sofreu alteração após a intervenção realizada, divergindo de algumas investigações encontradas ${ }^{4,8,22}$.

Um fator que pode ter interferido nesses resultados se refere à natureza unidimensional da intervenção implementada. Dada à multiplicidade de fatores que influenciam a estrutura mental de um indivíduo, há a necessidade de programas mais globais, com diferentes metodologias e com maior chance de atingir diversos aspectos psíquicos simultaneamente ${ }^{23}$. Aliado a isso, foi possível observar que o número reduzido de indivíduos que compunham os grupos de intervenção, bem como a manutenção de exercícios após a conclusão do programa pelos demais pesquisadores podem explicar, em parte, os resultados positivos em relação aos níveis de autoestima ${ }^{4}$, ansiedade ${ }^{16}$ e depressão ${ }^{6}$ dos universitários.

Uma recente revisão meta analítica reuniu evidências que apontaram efeitos positivos das abordagens não farmacológicas sobre sintomas depressivos e depressão em estudantes de enfermagem, sugerindo que estas intervenções podem servir como abordagens complementares e alternativas promissoras na redução dessas condições clínicas. As análises apontaram as intervenções de curto prazo (uma a oito semanas), de atenção plena e programas de controle do estresse como estratégias comuns e eficazes para melhora destes quadros ${ }^{21}$. Portanto, a inclusão de intervenções de manejo do estresse, ansiedade e depressão são imprescindíveis para o alcance positivo dessas condições, que se adotadas no presente estudo, poderiam ter gerado resultados mais satisfatórios na HADS.

Nota-se, ainda, que embora haja um número limitado de estudos, a autoeficácia foi melhorada neste e em outros programas voltados a promoção da saúde mental, sugerindo que esse recurso emocional é mais vulnerável a intervenções breves do que outros constructos, como a autoestima, e de sintomas clínicos, como a ansiedade e depressão, que podem estar mais enraizados, sofrendo influências de elementos que fogem do âmbito acadêmico, como traumas vividos desde a vida intraútero, passando por condições sociais e familiares precárias até situações de estresse pontuais e muito danosas, como perdas importantes. 
Embora com limitações, os resultados da presente pesquisa agregam dados científicos no repertório de possíveis intervenções que podem ser implementadas pela comunidade acadêmica, sendo de natureza inédita em âmbito nacional. Sugere um programa psicoeducacional, pautado em uma intervenção da NIC, que demonstrou validez no fortalecimento da percepção de autoeficácia, construto essencial na edificação de recursos psíquicos, uma vez que prepara o jovem para o enfrentamento assertivo do estresse pessoal e acadêmico, além de amortecer efeitos negativos de depressão e ansiedade.

As implicações do presente estudo incluem, ainda, um avanço no contexto da prevenção primária, já que abrange recursos emocionais positivos, em especial a autoeficácia, essenciais para prevenção da instalação de condições clínicas mentais negativas, em detrimento do predomínio de estudos voltados para estudantes com quadros de depressão e ansiedade já instalados.

Por fim, o programa psicoeducacional testado pode embasar o planejamento de estratégias preventivas adotadas pelas Universidades, podendo ser implementado a cada semestre, não de maneira pontual, mas de forma constante durante toda graduação.

\section{Limitações do estudo}

Algumas limitações devem ser consideradas. A amostra, apesar de ter sido recrutada de dois centros, foi restrita e, portanto, os resultados não podem ser generalizáveis. As variáveis relacionadas à saúde mental foram coletadas por meio de instrumento de autorrelato, o que pode ocasionar respostas inverídicas por parte dos participantes. Outro ponto significativo foi a não utilização de entrevista motivacional ou escala que avaliasse a prontidão para mudança na amostra pesquisada. Tais medidas poderiam ter norteado os pesquisadores na escolha de diferentes estratégias a ser empregadas.

Desta forma, recomenda-se que futuras pesquisas incluam o treinamento de outras habilidades, como manejo do estresse, ansiedade e depressão, relacionamentos interpessoais e enfrentamento, assim como outros parâmetros mais objetivos para avaliar a saúde mental.

\section{CONCLUSÃO}

O presente estudo demonstrou impacto positivo de um programa psicoeducacional nos níveis de percepção de autoeficácia em estudantes no início da graduação em enfermagem. Os níveis de autoestima, bem como os sintomas de ansiedade e depressão, não sofreram alterações significativas após a intervenção testada.

\section{REFERÊNCIAS}

1. Patton GC, Sawyer SM, Santelli JS, Ross DA, Afifi R, Allen NB, et al. Our future: a lancet commission on adolescent health and wellbeing. The lancet [Internet]. 2016 [cited 2020 Feb 20]; 387(10036):2423-78. DOI: http://dx.doi.org/10.1016/S01406736(16)00579-1.

2. Cuijpers P, Auerbach RP, Benjet C, Bruffaerts R, Ebert D, Karyotaki E, et al. The World Health Organization World Mental Health International College Student initiative: an overview. Int. J. Methods Psychiatr. Res. [Internet]. 2019 [cited 2020 Feb 20]; 28(2):e1761. DOI: http://dx.doi.org/10.1002/mpr.1761.

3. Rambod M, Sharif F, Khademian Z. The Impact of the preceptorship program on self-efficacy and learning outcomes in nursing students. Iran J. Nurs. Midwifery Res. [Internet]. 2018 [cited 2020 Feb 20]; 23(6):444-9. DOI: http://doi.org/10.4103/ijnmr.IJNMR_67_17.

4. Terp U, Hjarthag F, Bisholt B. Effects of a cognitive behavioral-based stress management program on stress management competency, self-efficacy and self-esteem experienced by nursing students. Nurse Educ. [Internet]. 2019 [cited 2020 Feb 20]; 44(1):E1-5. DOI: http://dx.doi.org/10.1097/NNE.0000000000000492.

5. Rayan A. Mindfulness, self-efficacy, and stress among final-year nursing students. J. Psychosoc. Nurs. Ment. Health Serv. [Internet]. 2019 [cited 2020 Feb 20]; 57(4):49-55. DOI: https://doi.org/10.3928/02793695-20181031-01.

6. Guo YF, Zhang X, Plummer V, Lam L, Cross W, Zhang JP. Positive psychotherapy for depression and self-efficacy in undergraduate nursing students: A randomized, controlled trial. Int. J. Ment. Health Nurs. [Internet]. 2017 [cited 2020 Feb 20]; 26(4):375-83. DOI: http://dx.doi.org/10.1111/inm.12255.

7. Hendriks T, Schotanus-Dijkstra M, Hassankhan A, Jong J, Bohlmeijer E. The efficacy of multi-component positive psychology interventions: a systematic review and meta-analysis of randomized controlled trials. J. Happiness Stud. [Internet]. 2020 [cited 2020 Jun 20]; 21:357-90. DOI: https://doi.org/10.1007/s10902-019-00082-1.

8. Ribeiro RM, Bragiola JVB, Eid LP, Ribeiro RCHM, Sequeira CAC, Pompeo DA. Impact of an intervention through Facebook to strengthen Self-esteem in nursing students. Rev. Latino Am. Enfermagem [Internet]. 2020 [cited 2020 Feb 20]; 28:e3237. DOI: https://doi.org/10.1590/1518-8345.3215.3237.

9. Souza I, Souza MA. Validação da escala de autoeficácia geral percebida. Rev. Univ. Rural [Internet]. 2004 [cited 2019 Apr 17]; 26(1-2):12-7. Available from:

https://www.researchgate.net/publication/260338439_Validacao_da_Escala_de_Autoeficacia_Geral_Percebida. 
10. Dini GM, Quaresma MR, Ferreira LM. Translation into Portuguese, Cultural Adaptation and Validation of the Rosenberg Selfesteem Scale. Rev. Bras. Cir. Plást. [Internet]. 2004 [cited 2019 Apr 17]; 19(1):41-52. Available from:

http://www.rbcp.org.br/details/322/en-US/translation-into-portuguese--cultural-adaptation-and-validation-of-the-rosenbergself-esteem-scale.

11. Francis B, Gill JS, Yit Han N, Petrus CF, Azhar FL, Ahmad Sabki Z, et al. Religious Coping, Religiosity, Depression and Anxiety among Medical Students in a Multi-Religious Setting. Int. J. Environ Res. Public Health [Internet]. 2019 [cited 2020 Feb 20]; 16(2):259. DOI: https://doi.org/10.3390/ijerph16020259.

12. Spadaro KC, Hunker DF. Exploring the effects of an online asynchronous mindfulness meditation intervention with nursing students on stress, mood, and cognition: a descriptive study. Nurse Educ. Today [Internet]. 2016 [cited 2020 Jun 20]; 39:163-9. DOI: https://doi.org/10.1016/j.nedt.2016.02.006.

13. Botega NJ, Bio MR, Zomignani MA, Garcia JC, Pereira WAB. Mood disorders among medical in-patients: a validation study of the hospital anxiety and depression scale (HAD). Rev. Saúde Pública [Internet]. 1995 [cited 2020 Jun 20]; 29(5):359-63. DOI: https://doi.org/10.1590/\$0034-89101995000500004.

14. Bandura A. Self-efficacy: Toward a unifying theory of behavioral change. Psychol. Rev. [Internet]. 1977 [cited 2020 Feb 20]; 84(2):191-215. DOI: https://doi.org/10.1037/0033-295X.84.2.191.

15. Bulecheck GM, Butcher HK, Dochterman JM, Wagner CM. NIC Classificação das intervenções de enfermagem. 6a ed. Rio de Janeiro: Editora Elsevier; 2016.

16. Brennan J, McGrady A, Lynch DJ, Schaefer P, Whearty K. A Stress Management Program for Higher Risk Medical Students: Preliminary Findings. Appl. Psychophysiol. Biofeedback [Internet]. 2016 [cited 2020 Jun 20]; 41(3):301-5. DOI: https://doi.org/10.1007/s10484-016-9333-1.

17. Li C, Yin H, Zhao J, Shang B, Hu M, Zhang P, et al. Interventions to promote mental health in nursing students: a systematic review and meta-analysis of randomized controlled trials. J. Adv. Nurs. [Internet]. 2018 [cited 2020 Jun 20]; 74(12):2727-41. DOI: https://doi.org/10.1111/jan.13808.

18. Barbaranelli C, Paciello M, Biagioli V, Fida R, Tramontano C. Positivity and behaviour: the mediating role of self-efficacy in organisational and educational settings. J. Happiness Stud. [Internet]. 2019 [cited 2020 Jun 20]; 20:707-27. DOI: https://doi.org/10.1007/s10902-018-9972-4.

19. Pérez-Fuentes MDC, Jurado MDMM, Gázquez Linares JJ. Explanatory value of general self-efficacy, empathy and emotional intelligence in overall self-esteem of healthcare professionals. Soc. Work Public Health [Internet]. 2019 [cited 2020 Jun 20]; 34(4):318-29. DOI: https://doi.org/10.1080/19371918.2019.1606752.

20. Recabarren RE, Gaillard C, Guillod M, Martin-Soelch C. Short-term effects of a multidimensional stress prevention program on quality of life, well-being and psychological resources. A randomized controlled trial. Front Psychiatry [Internet]. 2019 [cited 2020 Jun 20]; 10:88. DOI: https://doi.org/10.3389/fpsyt.2019.00088.

21. Chen D, Sun W, Liu N, Wang J, Guo P, Zhang X, et al. Effects of nonpharmacological interventions on depressive symptoms and depression among nursing students: a systematic review and meta-analysis. Complement. Ther. Clin. Pract. [Internet]. 2019 [cited 2020 Jun 20]; 34:217-28. DOI: https://doi.org/10.1016/j.ctcp.2018.12.011.

22. Choi YJ. Evaluation of a program on self-esteem and ego-identity for Korean nursing students. Nurs. Health Sci. [Internet]. 2016 [cited 2020 Jun 20]; 18(3):387-92. DOI: https://doi.org/10.1111/nhs.12281.

23. Frajerman $A$. Which interventions improve the well-being of medical students? A review of the literature. Encephale [Internet]. 2020 [cited 2020 Jun 20]; 46(1):55-64. DOI: https://doi.org/10.1016/j.encep.2019.09.004. 